\title{
Colloids as model systems for liquid undercooled metals
}

\author{
Patrick Wette, ${ }^{1}$ Ina Klassen, ${ }^{1}$ Dirk Holland-Moritz, ${ }^{1}$ Thomas Palberg, ${ }^{2}$ Stephan V. Roth, ${ }^{3}$ and Dieter M. Herlach ${ }^{1, *}$ \\ ${ }^{1}$ Institut für Materialphysik im Weltraum, Deutsches Zentrum für Luft- und Raumfahrt, 51170 Köln, Germany \\ ${ }^{2}$ Institut für Physik, Johannes-Gutenberg-Universität Mainz, 55128 Mainz, Germany \\ ${ }^{3}$ HASYLAB, DESY, Notkestrasse 85, 22603 Hamburg, Germany
}

(Received 18 July 2008; published 27 January 2009)

\begin{abstract}
Charged colloidal particles interact via a hard core Yukawa potential, while isotropic Lennard-Jones-like potentials are frequently used as pair potentials in metals. We present measurements of the structure factor of shear molten monodisperse colloids and molten metals using ultrasmall-angle x-ray scattering and elastic neutron scattering, respectively. In both systems data analysis gives evidence of fivefold-symmetric short-range order becoming more pronounced with increasing deviations from equilibrium. The experiments demonstrate that in both systems topological effects control ordering in the melt state.
\end{abstract}

DOI: 10.1103/PhysRevE.79.010501

PACS number(s): 61.20.-p, 47.57.J-

Metals are nontransparent systems with atoms, which show fast relaxation dynamics in the liquid state. Therefore, it is extremely difficult to directly observe, e.g., solid-liquid interfaces, nucleation of crystalline phases, and instabilities of a solidification front. In contrast, colloidal suspensions are transparent and the dynamics of their particles, ranging in size from $10 \mathrm{~nm}$ to $10 \mu \mathrm{m}$, is much more sluggish than the relaxation of atoms in metals. Detailed investigations of phase transitions are possible in colloids [1] even in real space [2]. Therefore, colloidal systems with tunable interactions are often discussed as model systems for atomic materials [3]. In the present work, we indeed demonstrate that charged sphere colloidal suspensions are valuable model systems for metals.

A knowledge of short-range order in a liquid is of fundamental importance for understanding the nucleation of solid phases. The first attempt to compare the short-range order of alkali metals like $\mathrm{Rb}, \mathrm{K}$, and $\mathrm{Cs}$ in the liquid state and colloidal fluids under equilibrium conditions was reported by Cotter and Clark [4]. The present work addresses the shortrange order far from equilibrium in the melt phase both of colloidal systems and of pure metals. Deviations from equilibrium are measured by the difference of particle densities of metastable and stable liquids in colloidal suspensions and by the undercooling below the melting temperature of metals, respectively. For pure metallic melts with compact local order and isotropic bonding, Frank [5] hypothesized an icosahedral short-range order independent of the structure of the corresponding solid phases. Surrounding a central spherical atom by an icosahedral shell of 12 nearest neighbors of the same size results in the locally densest packing. If a second shell of atoms is arranged onto the tetrahedral surfaces of an icosahedron, a dodecahedron is formed consisting of 33 atoms. Frank's hypothesis was used to explain the large undercoolings of pure metallic melts observed by Turnbull [6] such that the fivefold symmetry of icosahedral shortrange order must be broken before a crystal with its translational symmetry can be formed.

Experimental evidence of icosahedral short-range order in

*Corresponding author. dieter.herlach@dlr.de liquid metals came from x-ray and neutron diffraction and $\mathrm{X}$-ray absorption spectroscopy investigations [7-10]. In computer simulations of metastable Lennard-Jones (LJ) liquids also an icosahedral short-range order was found that increases with rising undercooling [11]. A fraction of $61 \%$ of atoms are organized within an icosahedral environment that is expected to be even larger in the bulk of real LJ liquids like metals [12]. In hard sphere simulations, on the other hand, groups of 13 atoms do not form icosahedra [13]. Moreover, for layers of fluid hard sphere systems close to a crystalline substrate, no icosahedral short-range order was detected [14]. Using confocal microscopy, the structure of a colloidal melt of very weakly charged hard spheres was investigated. While some particles were found in surroundings with cubic or hexagonal close-packed character, most particles are arranged in local structures that contain fragments of icosahedra or even full icosahedra. The icosahedral character increased with volume fraction [15].

In contrast to these topological considerations, it was suggested that in metallic systems the electrons control structure formation. Indirect ion-ion interaction causes an oscillatory effective pair potential due to the electric polarizability of the electron cloud around each ion screening its local charge. The oscillations are known as Friedel oscillations [16]. A matching of ionic positions at short- and medium-range distances with the minima of the effective pair potential leads to a lowering of the energetic states of the respective ions and will stabilize the corresponding structural state. It was argued that such effects influence the structure formation in liquids and glasses [17]. Since electrons are not present in structure formation in colloidal systems, ordering has to be by topological processes only.

In the present Rapid Communication we address both the question of the influence of the interaction potential and the origin of the fivefold-symmetric order. We present experimental investigations on metallic systems with a LJ-like potential and charged colloidal suspensions interacting via a hard sphere Yukawa repulsion. In the latter case, formation of a supercritical fluid state and crystallization are induced at constant volume via an increased particle density and an increased particle charge. Nonequilibrium melts, on the other hand, are obtained by shear melting [18].

The structure factor of pure liquid metals was measured 


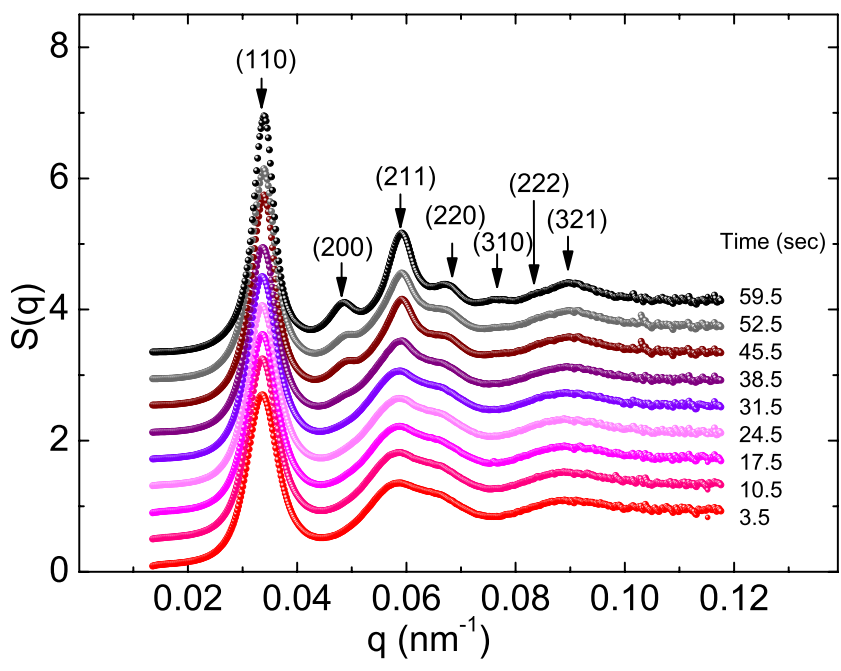

FIG. 1. (Color online) Static structure factors $S(q)$ at different times for a colloidal suspension at $n=113 \mu \mathrm{m}^{-3}$ and maximum interaction that was shear melted (lower curve) and subsequently crystallizes into a bcc structure (upper curve). $S(q)$ are shifted for clarity; time increases from bottom to top. Miller indices are indicated, clearly identifying the bcc structure of the polycrystalline solid formed.

by neutron scattering [9] and by x-ray scattering using synchrotron radiation [10] on levitation-processed metals in the stable and undercooled liquid state. In the colloidal system, with their 3-4 orders of magnitudes larger particle sizes, the structure was determined by ultrasmall-angle x-ray scattering (USAXS). To analyze the measured structure factor with respect to short-range ordering the same formalism [19] was utilized for metallic and colloidal systems.

We used colloidal silica particles (diameter $84 \pm 6 \mathrm{~nm}$ ) synthezised by Stöber synthesis [20]. The particles carry weakly acidic silanol groups ( $\mathrm{Si}-\mathrm{OH})$ on their surfaces, which partly dissociate in a deionized water environment. The surface charge density of the particles may be increased by adding sodium hydroxide $(\mathrm{NaOH})$ [21], leading to the reaction $\mathrm{SiOH}+\mathrm{NaOH} \rightarrow \mathrm{SiO}^{-}+\mathrm{Na}^{+}+\mathrm{H}_{2} \mathrm{O}$, until all surface groups are dissociated, corresponding to a maximum effective interaction. Further addition of $\mathrm{NaOH}$ leads to a screening effect, which subsequently reduces the interaction between the particles. For aqueous silica dispersions, the particle number density $n$ and the $\mathrm{NaOH}$ concentration $c_{\mathrm{NaOH}}$ are the control parameters for the screened Coulomb interaction. From shear modulus measurements [22] the effective particle charge at maximum interaction conditions was found to be independent of $n$ at a value of $Z_{\text {eff }}=340 \pm 20$. We carefully checked that the charge was not altered by exposure to $\mathrm{x}$ rays. The typical phase diagram shows reentrant melting as a function of $c_{\mathrm{NaOH}}$ with the crystalline region becoming broader with increasing $n$. For the present measurements on the melt state, an external peristaltic pump shear melts the colloidal suspension. After cessation of shear the suspension is in a metastable state of fluid order, equivalent to the undercooled state of a metallic melt.

The USAXS experiments were carried out at the beamline BW4 at HASYLAB (DESY) [23]. The accessible scattering vectors $q$ range from 0.008 to $0.280 \mathrm{~nm}^{-1}$ at a working dis-

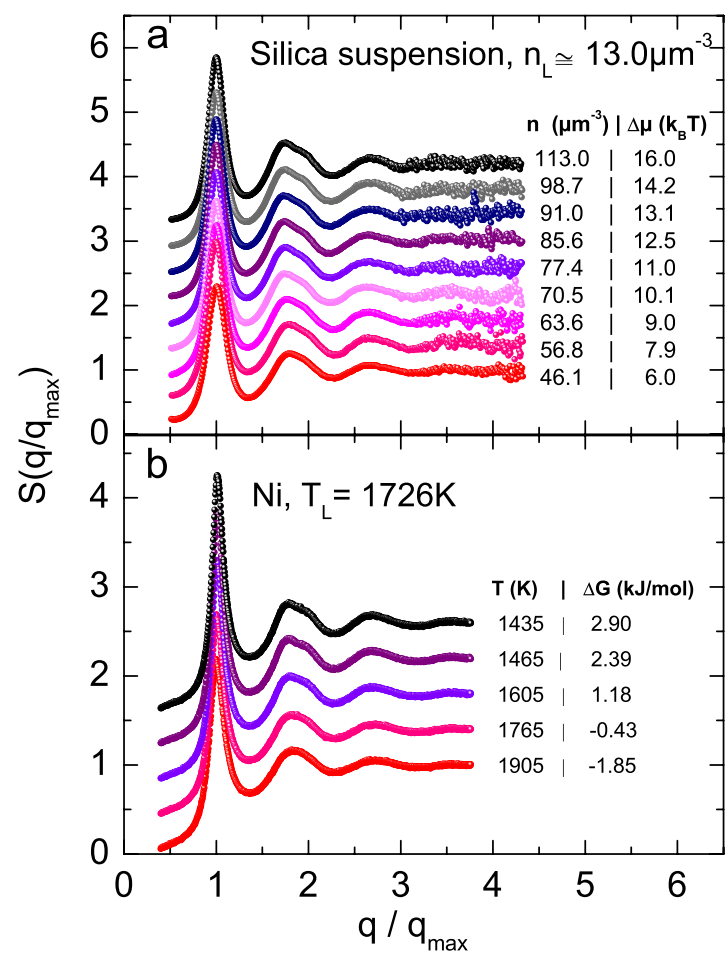

FIG. 2. (Color online) Structure factor $S\left(q / q_{\max }\right)$ as a function of normalized scattering vector $q / q_{\max }$, measured on the melt phase of a colloidal suspension at maximum interaction by USAXS (a) and measured on liquid Ni by neutron scattering (b). For the two physically different systems the same behavior is observed. A shoulder appears in the second diffraction peak, which systematically becomes more pronounced with increasing metastability, i.e., increasing chemical potential difference for colloids, $\Delta \mu$, and increasing driving force, $\Delta G$, for metals.

tance of $13.3 \mathrm{~m}$ with a resolution of $2048 \times 2048$ (pixel size $79.1 \mu \mathrm{m})$. The incident wave length is $\lambda=0.138 \mathrm{~nm}$. The USAXS technique enables measurements of time-resolved diffraction patterns $I(q)=I_{0} P(q) S(q)$ of colloidal suspensions. The X-ray intensity $I_{0}$ was measured with a standard Lupolen sample. The particle form factor $P(q)$ is accessible at low $n$ and large $c_{\mathrm{NaOH}}$, where particle interactions are sufficiently suppressed and a disordered state $[S(q)=1]$ results. $P(q)$ corresponds to the atomic form factor in metallic systems. For the measured intensity $I(q)$, a conventional background and transmission correction was carried out. Figure 1 shows the time evolution of the structure factor of the colloidal system from the nonequilibrium liquid state (lower curve) to the stable solid phase (upper curve) at a particle number density $n=113 \mu \mathrm{m}^{-3}$. The time interval between each curve corresponds to $7 \mathrm{~s}$ due to the integration interval of $3.5 \mathrm{~s}$ and the detector readout time of $3.5 \mathrm{~s}$ Figure $1 \mathrm{dem}-$ onstrates that at lower times $(<25 \mathrm{~s})$ there is no change of the structure factor. This assures that especially the first structure factor taken after $3.5 \mathrm{~s}$ corresponds to a metastable melt state. For longer times a structural transition into a stable bcc phase is observed. This behavior is representative for the analyzed system over the accessible range of particle concentrations at maximum interaction.

Figure 2 compares experimental results for the structure 
factors of the nonequilibrium liquid state for a colloidal system measured by USAXS (a) with results of the experimentally determined structure factors of undercooled metallic melt previously measured by neutron scattering on liquid $\mathrm{Ni}$ (b) [9]. In order to compare directly the structure factors of the colloidal suspension and the metal, $S\left(q / q_{\max }\right)$ is plotted as a function of dimensionless wave vectors $q / q_{\max }$ with $q_{\max }$ the wave vector at the first maximum of $S(q)$. The deviations from equilibrium for the colloidal suspension are given by the chemical potential difference between the metastable and stable fluids, $\Delta \mu$. We here followed Aastuen et al. [24] and determined $\Delta \mu$ by measuring the growth velocities of colloidal crystals in dependence on the particle number density $n$ by means of microscopy. The growth velocity obeys a Wilson-Frenkel behavior $v=v_{0}\left[1-\exp \left(\Delta \mu / k_{B} T\right)\right]$, where $v_{0}$ is the limiting velocity and $\Delta \mu / k_{B} T=B\left(n-n_{L}\right) / n_{L}$, with the fit parameter $B$ and the particle number density at equilibrium $n_{L}=13.0 \mu \mathrm{m}^{-3}$. Corresponding driving forces in metallic systems can be determined via the Gibbs free energy difference $\Delta G=G_{\text {solid }}-G_{\text {liquid }} \propto \Delta \mu$, which is estimated by the linear approximation $\Delta G=\Delta S_{f}\left(T_{L}-T\right)$ with $\Delta S_{f}$ as the heat of fusion and $T_{L}$ the melting temperature.

The diffraction measurements are strikingly similar and show up to four oscillations with decreasing intensity if $q / q_{\max }$ is increasing. In both systems the experiments reveal an asymmetric second oscillation of $S\left(q / q_{\max }\right)$ with a shoulder that becomes more pronounced with increasing deviations from thermodynamic equilibrium. The shoulder position corresponds to the length scale between particles surrounding a central one in a fivefold-symmetric way. The shoulder can therefore be considered as an indication for icosahedral short-range order to be present in both physically different systems [25].

The appearance of a shoulder cannot be reproduced by simulations of the structure factor of a simple hard sphere system [26]. Integral equation approaches for hard sphere Yukawa fluids under equilibrium conditions also do not show this feature [27]. We therefore took the approximation proposed by [19] for $S\left(q / q_{\max }\right)$ for both the colloid and the metal. The simulations assume the liquid to be composed of isolated structural units and neglect intercorrelations of these units. Body centered cubic (bcc), face centered cubic (fcc), or equivalently hexagonal close-packed (hcp) clusters as well as perfect icosahedral and dodecahedral clusters were considered. This certainly neglects the wealth of imperfect clusters of fivefold symmetry [28] which are also likely to be present, but it is sufficient to unequivocally support our main conclusions. Use of isolated clusters instead of a continuous structure results in low amplitudes of the principal peak. Hence a three-parameter fit to the remaining three oscillations was performed, optimizing the shortest mean distance $r_{0}$ of the particles, its mean thermal variation $\delta r_{0}^{2}$, and the fraction of atoms organized in each structural unit, $c$. The simulated structure factors of the colloidal suspension and the metallic melt are compared with the experimental results in Fig. 3. The shoulder feature of the colloidal melt is somewhat less pronounced than for $n=113 \mu \mathrm{m}^{-3}$, but even more closely resembles that of the molten metal. This is also reflected in the fit results.

Suppose that a bcc-like short-range order of the under-

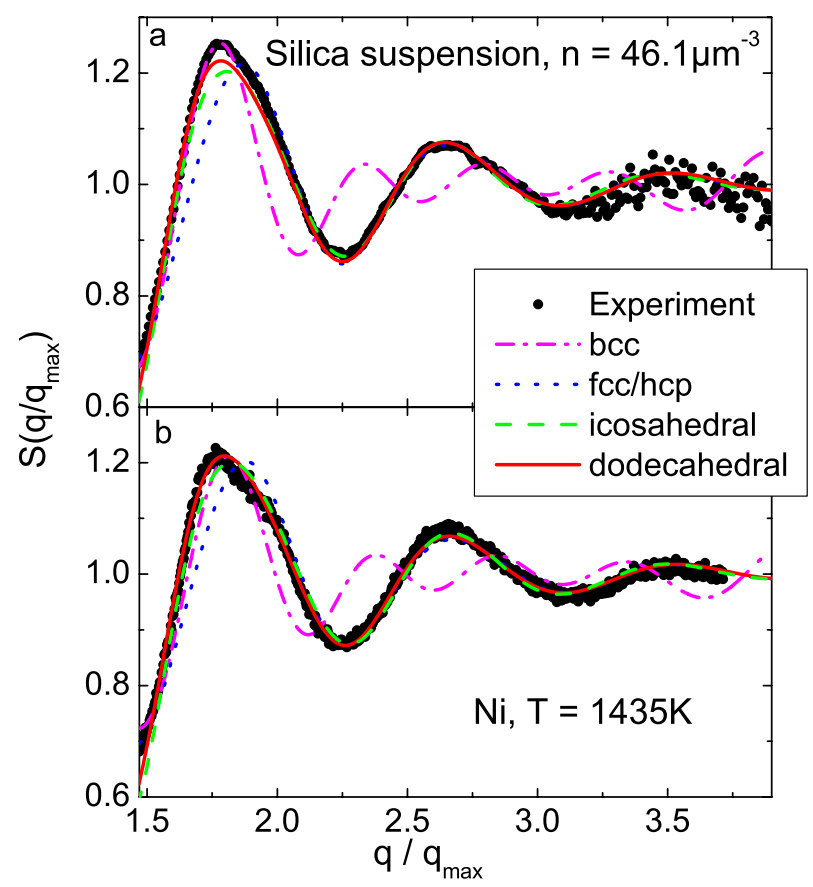

FIG. 3. (Color online) Structure factors $S\left(q / q_{\max }\right)$ of a colloidal melt with $n=46.1 \mu \mathrm{m}^{-3}$ (a) and a Ni melt with $T=1435 \mathrm{~K}$ (b). Measured data are represented by symbols and lines show results of simulations assuming short-range order with different symmetries prevailing in the melt: bcc (dash dotted), fcc and hcp (dotted), icosahedral (dashed), and dodecahedral (solid).

cooled melt utterly fails in describing the data. A fcc-type short-range order performs better but does not agree with the shape and peak positions in the second oscillation, where the significant asymmetry of a shoulder is observed. An agreement is achieved if icosahedral units of fivefold symmetry are assumed. It becomes even better if larger dodecahedral aggregates are assumed. For the colloidal suspension, the best fit of the simulations to the experiments for icosahedral ordering gives the values: $r_{0}=315 \mathrm{~nm}, \delta r_{0}^{2}=487 \mathrm{~nm}^{2}$, and $c$ $=99 \%$. A dodecahedral type of ordering shows the related parameters as $r_{0}=319 \mathrm{~nm}, \delta r_{0}^{2}=435 \mathrm{~nm}^{2}$, and $c=99 \%$. By comparison, for the simulation of liquid $\mathrm{Ni}$ we obtain $r_{0}$ $=0.238 \mathrm{~nm}, \delta r_{0}^{2}=0.29 \times 10^{-3} \mathrm{~nm}^{2}$, and $c=99 \%$ (icosahedral) and $r_{0}=0.242 \mathrm{~nm}, \quad \delta r_{0}^{2}=0.26 \times 10^{-3} \mathrm{~nm}^{2}, \quad$ and $c=95 \%$ (dodecahedral).

To summarize the results, comparison of the structure factors $S\left(q / q_{\max }\right)$ of a colloidal melt and of molten Ni showed strikingly similar results if scaled with the scattering vector corresponding to the nearest neighbor distance. In particular, for both these physically different systems an asymmetric shoulder in the second maximum of $S\left(q / q_{\max }\right)$ was observed, which became more pronounced with increasing deviations from equilibrium. Hence, low-salt hard sphere Yukawa interactions are found suitable to model LJ-like interactions in metallic systems. Moreover, the experimental data were analyzed by an approach assuming isolated structural units in the liquid. The simulations demonstrate that the assumption of bcc, fcc, or hcp structural units does not describe the experimental results. The agreement between simulation and experiments becomes nearly quantitative when icosahedral 
or dodecahedral clusters are assumed. Given the simplicity of the model calculations made, we as yet may not insist on the presence of perfect icosahedra or dodecahedra. But we may safely conclude that in both metastable melts fivefoldsymmetric local environs are abundant. Given the presence of this striking similarity despite the different types of interactions in the two systems, we further suggest that the slightly soft repulsive part of both the LJ-like potential of liquid metals and the hard core Yukawa potential of the charged spheres is a precondition for the formation of icosahedral short-range order as hypothesized earlier [12]. The finding that the repulsive term of the interaction potential is decisive for the formation of the short-range order in liquids is in full agreement with previous theoretical investigations [29]. Since electronic effects are absent in colloidal systems, the similarity of the structure factors of the colloidal and the metallic melts suggests that short-range order is controlled by topological effects in both these physically different systems. We have identified a colloidal system consisting of charged silica particles in a salty solution that behaves as a metal concerning the formation of short-range order in the metastable liquid state. This finding is of general importance since short-range order essentially governs the formation of solid-liquid interfaces and crystal nucleation in undercooled melts [30].

We thank N. Lorenz, S. Klein, and A. Timmann for experimental support, M. Sperl, H. J. Schöpe, and J. Horbach for discussions, H. Reiber for sample preparation, and A. Meyer for support. Financing by DFG within Grants No. HE1601/23, No. HE1601/24, No. Pa459/14, and No. $\mathrm{Pa} 459 / 16$ is gratefully acknowledged.
[1] V. J. Anderson and H. N. W. Lekkerkerker, Nature (London) 416, 811 (2002).

[2] U. Gasser et al., Science 292, 258 (2001).

[3] A. Yethiraj, Soft Matter 3, 1099 (2007).

[4] L. K. Cotter and N. A. Clark, J. Chem. Phys. 86, 6616 (1987).

[5] F. C. Frank, Proc. R. Soc. London, Ser. A 215, 43 (1952).

[6] D. Turnbull, J. Chem. Phys. 20, 411 (1952).

[7] H. Reichert et al., Nature (London) 408, 839 (2000).

[8] A. Filipponi, A. Di Cicco, and S. De Panfilis, Phys. Rev. Lett. 83, 560 (1999); A. Di Cicco, A. Trapananti, S. Faggioni, and A. Filipponi, ibid. 91, 135505 (2003).

[9] T. Schenk, D. Holland-Moritz, V. Simonet, R. Bellissent, and D. M. Herlach, Phys. Rev. Lett. 89, 075507 (2002).

[10] K. F. Kelton et al., Phys. Rev. Lett. 90, 195504 (2003).

[11] F. Yonezawa, in Solid State Phys., edited by H. Ehrenreich and D. Turnbull (Academic Press, London, 1991) Vol. 45, p. 179.

[12] H. Jónsson and H. C. Andersen, Phys. Rev. Lett. 60, 2295 (1988).

[13] N. J. A. Sloane et al., Discrete Comput. Geom. 14, 237 (1995).

[14] M. Heni and H. Löwen, Phys. Rev. E 65, 021501 (2002).

[15] U. Gasser, A. Schofield, and D. A. Weitz, J. Phys.: Condens. Matter 15, S375 (2003).

[16] J. Hafner, Mater. Sci. Eng., A 178, 1 (1994).

[17] P. Häussler, Phys. Rep. 222, 65 (1992).
[18] B. J. Ackerson, N. A. Clark, Physica A 118, 221 (1983).

[19] V. Simonet et al., Phys. Rev. B 58, 6273 (1998); V. Simonet, F. Hippert, M. Audier, and R. Bellissent, ibid. 65, 024203 (2001)

[20] W. Stöber, A. Fink, and E. Bohn, J. Colloid Interface Sci. 26, 62 (1968)

[21] J. Yamanaka, Y. Hayashi, N. Ise, and T. Yamaguchi, Phys. Rev. E 55, 3028 (1997).

[22] P. Wette, H. J. Schöpe, and T. Palberg, J. Chem. Phys. 116, 10981 (2002).

[23] S. V. Roth et al., Rev. Sci. Instrum. 77, 085106 (2006).

[24] D. J. W. Aastuen, N. A. Clark, L. K. Cotter, and B. J. Ackerson, Phys. Rev. Lett. 57, 1733 (1986).

[25] D. R. Nelson and F. Spaepen, in Solid State Phys., edited by H. Ehrenreich and D. Turnbull (Academic Press, New York, 1989), Vol. 42, p. 190.

[26] T. Schenk, Ph.D. thesis, Ruhr-University Bochum, 2002.

[27] G. Nägele, Phys. Rep. 272, 217 (1996).

[28] J. P. K. Doye, D. J. Wales, and R. S. Berry, J. Chem. Phys. 103, 4234 (1995).

[29] J. D. Weeks, D. Chandler, and H. C. Andersen, J. Chem. Phys. 54, 5237 (1971).

[30] K. F. Kelton, A. L. Greer, D. M. Herlach, and D. HollandMoritz, MRS Bull. 29, 940 (2004). 